\title{
Brainstem auditory evoked potentials for intraoperative neurophysiological monitoring
}

\author{
Nishanth Sampath, Sudhakar Subramaniam, Vijay Sankaran, Roopesh Kumar, Suresh Bapu KR
}

\section{BRAINSTEM AUDITORY EVOKED POTENTIALS}

Spontaneous electrical activity of brain electroencephalogram (EEG) - is of significant amplitude $(\sim 100 \mu \mathrm{V})$ and can mask the small electrical activities produced by stimulation of a specific sensory modality. The strategy to extract electrical activity produced by the auditory system alone $(<1 \mu \mathrm{V})$ is to provide repetitive auditory stimuli and to average the resulting electrical responses recorded from the scalp, so that time-locked events alone would stand out, with cancellation of random noise, which in this case is background EEG.

There are critical differences in recording of brainstem auditory evoked potentials (BAEPs) in outpatient versus intra-operative setting. Whereas standard stimulation and recording protocol, normative data and noise-free ambience in sound-proof rooms are available for outpatient recording, intraoperative technique has to be optimised for each patient so that good electrophysiological signals are obtained. Baseline obtained just after induction serves as control for comparison. Anaesthesia, temperature, blood pressure and local perfusion all affect the recorded signals. ${ }^{[1]}$ Drilling of bone, use of cautery and cavitron ultrasonic surgical aspirator (CUSA) can contaminate the recordings. ${ }^{[2]}$ All these have to be managed inside the hostile electrical environment of operating room. A good communication between the operating surgeon, anaesthesiologist and intraoperative neurophysiologist is bare minimum to obtain reliable recordings.

\section{Stimulation}

Delivery of auditory stimulus is by conformable ear foams connected to transducer by means of plastic

Institute of Neurosciences, SRM Institutes of Medical

Science Hospital, Chennai, Tamil Nadu, India

\begin{tabular}{|l|l|}
\hline \multicolumn{2}{|c|}{ Access this article online } \\
\hline Quick Response Code: & Website: \\
\hline & www.jnaccjournal.org \\
\cline { 2 - 2 } & \\
\hline
\end{tabular}

tubing. It should be noted that introduction of plastic tube adds to delay in recorded waveforms in proportion to the tube length (usually $0.88 \mathrm{~ms}$ ). ${ }^{[3]}$ Broadband clicks of $100 \mu$ s duration and of alternating polarity are used with a repetition rate roughly in the range between 10 and $30 / \mathrm{s}$. Care should be taken that rate is not a factor of line frequency $(50 \mathrm{~Hz})$. Stimulation is at an intensity of 100-110 dB sound pressure level to the ear whose function is to be preserved. The other ear should be masked with white noise 30-40 dB less than the stimulation intensity so as to prevent recording responses by stimulation of contralateral ear. In case of large tumours with non-serviceable hearing ipsilateral to the side of surgery, monitoring has to be done for contralateral ear. Hearing assessment with audiometry and speech discrimination score is indicated in all patients in whom intraoperative hearing preservation is attempted. Higher stimulus intensity has to be used if there is a pre-operative hearing loss. Care should be taken that ear is clear of wax prior to induction.

The following are a few measures to ensure adequate stimulus delivery:

- The plastic tubing is not kinked

- The ear foam is not dislodged

- Securing the ear with adhesive tape so that irrigating fluid/blood does not enter the external auditory canal.

\section{Recording}

Standard EEG disc electrodes (after skin preparation and jelly application) or sub-dermal needle electrodes are used for recording. Impedances have to be below 5 kohms. At least 2 channels are used Cz-Ai and Cz-Ac (10-20 electrode system). The third channel of Ai-Ac can also be used. Ground is placed over Fpz. A number of

This is an open access article distributed under the terms of the Creative Commons Attribution-NonCommercial-ShareAlike 3.0 License, which allows others to remix, tweak, and build upon the work non-commercially, as long as the author is credited and the new creations are licensed under the identical terms.

For reprints contact: reprints@medknow.com

How to cite this article: Sampath N, Subramaniam S, Sankaran V, Kumar R, Suresh Bapu KR. Brainstem auditory evoked potentials for intraoperative neurophysiological monitoring. J Neuroanaesthesiol Crit Care 2016;3:1-3. 
trials range from 500 to 2000 however this can be tailored according to signal-to-noise ratio (SNR) so that feedback could be given to the surgeon at the earliest. Analysis time can be kept between 15 and $20 \mathrm{~ms}$ from stimulus onset. Filter settings of $50-150 \mathrm{~Hz}$ for high-passing and $1000-3000 \mathrm{~Hz}$ for low-passing can be used as the primary spectral energy is between 50 and $1000 \mathrm{~Hz}$. These settings could be altered according to the context; use of notch filter is permissible, as long as filter settings are the same throughout monitoring. Modern amplifiers permit pausing averaging when an artefact as defined by an amplitude criterion is encountered.

\section{Waveform characteristics, measurements and generators}

Jewett et al. first described BAEPs as series of scalp-recorded potentials obtained by activation of auditory neural pathways. ${ }^{[4]}$ He used Roman letters I - VII to describe the peaks obtained in BAEP recordings. BAEP monitoring for surgeries came into vogue in the late 1970s. ${ }^{[5]}$ Typical BAEPs are shown in Figure 1.

Wave I has a typical post-stimulus latency of $\leq 2 \mathrm{~ms}$. Waves II - V occur roughly at $1 \mathrm{~ms}$ intervals after wave I. Waves I, III and V are more stable within and across subjects. Since they are far-field potentials, they are of low amplitudes $(\sim 0.5 \mu \mathrm{V})$. Waves I and V are most useful for intraoperative neurophysiological monitoring (IONM), followed by waves II and III. Waves IV, VI and VII are of minimal use. ${ }^{[1]}$

Knowledge of generators of various BAEP components can help in correlating the BAEP changes with possible site (s) and mechanisms of injury. ${ }^{[3]}$ Wave I is generated by action potential volleys from the most distal part of auditory nerve (AN). All subsequent components have multiple generators, but for purposes of clinical interpretation, can be assumed to be originating predominantly from a specific site of auditory pathway. Wave II has contributions from both the proximal AN and cochlear nucleus. The putative generator of wave III is superior olivary complex (lower pons). Wave IV is inconsistent and many times merged with wave $\mathrm{V}$ and is speculated to arise from middle pons in the lateral lemniscus. Wave $\mathrm{V}$ generator is corroborated to arise from upper pons/lower midbrain at the level of inferior colliculus. Wave VI is considered to originate from medial geniculate body and wave VII from auditory radiations.

With the help of changes in absolute latencies of individual waves, inter-peak latencies of I-III, III-V and $\mathrm{I}-\mathrm{V}$ and absolute amplitudes, it is possible to localise the possible site of injury and to corroborate these findings within the context of the surgical step. Serial tracking of the waveforms in stack window can help appreciate these changes quickly.

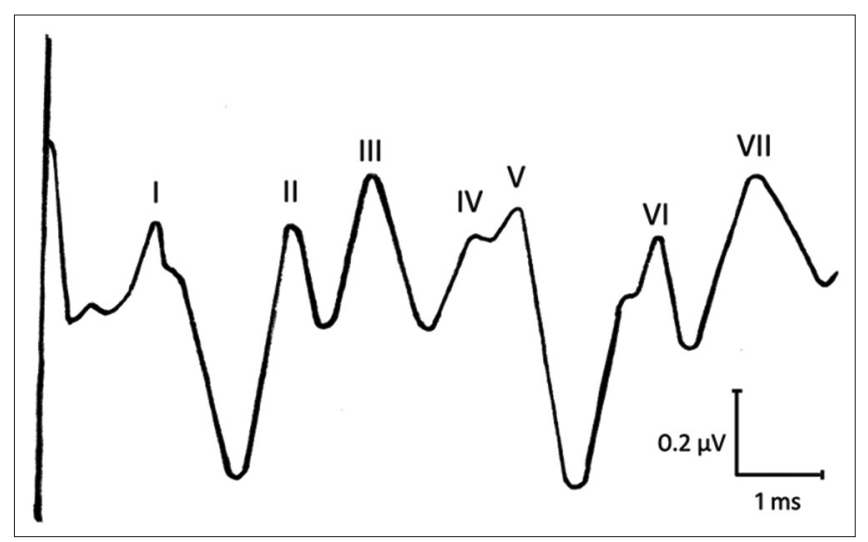

Figure 1: Schematic illustration of typical brainstem auditory evoked potentials waveforms

\section{Criteria for warning}

Typical (however, arbitrary) criteria for alerting the surgeon is $1 \mathrm{~ms}$ latency prolongation or $50 \%$ drop in amplitude of wave V. However, in the light of new evidence, it would be prudent to develop criteria according to surgical procedure. ${ }^{[6]}$ Other waves (I and III) and other milder changes in wave $\mathrm{V}$, if consistent and related to the surgical step, should be intimated to the surgeon, so that prompt measures may be taken to reverse or prevent worsening of electrophysiological findings.

\section{Mechanisms of injury}

The mechanisms of injury to auditory pathways during surgery can be classified as mechanical (severing, avulsion, compression and stretching), ischaemia (damage to vessels/vasospasm) or thermal (heat injury from cautery).

\section{Surgical correlate}

BAEP monitoring is indicated in the following surgeries: Acoustic neuroma, other cerebellopontine angle and fourth ventricular tumours, microvascular decompression of V, VII, VIII and IX cranial nerves, brainstem tumours, vascular surgeries of posterior cerebral circulation, sub-occipital decompression and skull base surgeries.

The surgeon and the neurophysiologist have to be aware that the risk to auditory pathways is maximal ${ }^{[2]}$ during the following surgical steps: Direct mechanical damage to cochlea or labyrinth while drilling of temporal bone, damage to internal auditory artery causing cochlear ischaemia/infarction, cerebellar retraction causing traction to VIII nerve, avulsion of the nerve when attempting complete tumour resection, direct mechanical damage caused by use of CUSA and ischaemic insult during posterior fossa vascular injury. BAEP changes have also been reported during dural closure as a result of shift of contents of posterior fossa, ${ }^{[7]}$ and monitoring has to be continued during closure. 


\section{ELECTROCOCHLEOGRAM}

The technique consists of placing the active electrode close the cochlea (usually in the proximity of tympanic membrane). The N1 response corresponds to wave I of BAEP. Being a near-field response, it is of much larger amplitude $(2-20 \mu \mathrm{V})$ and takes less time to average and gives a faster feedback than BAEP. However, it reflects the activity of the most distal part of AN and is not useful in assessing the more proximal pathways.

\section{AUDITORY NERVE COMPOUND ACTION POTENTIAL}

Direct recording from AN may be attempted if it is feasible for the surgeon to get access to the proximal AN. Special electrodes (e.g. Cueva electrode) are available for this purpose. Bipolar electrodes are available to distinguish nerve from tumour tissue. The largest responses $(\sim 50 \mu \mathrm{V})$ are produced in this technique and hence, the least time is required for averaging.

\section{Special considerations}

Among the various electrophysiological signals monitored during IONM, BAEPs, electrocochleogram (ECochG) and AN compound action potential (AN-CAP) are most resistant to anaesthetic effects. Inhalational agents do cause minor changes in amplitude and latencies of BAEP, but since the baseline obtained is already under the surgical levels of anaesthesia, further changes in anaesthetic regime hardly matter. ${ }^{[2]}$ However, hypothermia does markedly affect the signals.

Large artefacts are produced during drilling, use of CUSA and cautery that SNR would be very low. If they coincide with the critical steps of surgery, it is prudent to request the surgeon to pause the use of these devices giving time for signals to get averaged, so that critical changes are not missed.

\section{APPLICATIONS}

The goal of monitoring is hearing preservation in patients with small tumours and serviceable hearing. However, in case of large tumours, hearing may already be lost; in such cases, monitoring of BAEPs can still help in preserving brainstem function. Monitoring can also be used to document intraoperative restoration of hearing. ${ }^{[3]}$

\section{CONCLUSION}

Monitoring of auditory pathways is useful to preserve hearing and monitor brainstem function. Combining the techniques of BAEP, ECochG and AN-CAP can improve the predictive value of neurological outcome. Various technical factors need to be kept in mind to obtain good signals. The surgeon and the neurophysiologist must be aware of critical surgical steps that can damage the auditory pathways. Anaesthesiologist must make sure that the cerebral perfusion and body temperature are maintained during the procedure for effective monitoring.

\section{Acknowledgements}

The authors sincerely acknowledge the efforts of Dr. Indu and Mr. Raj for their assistance in preparing the BAEP illustration and of Ms. Agnes Philomina for proof-reading the manuscript.

\section{Financial support and sponsorship}

Nil.

\section{Conflicts of interest}

There are no conflicts of interest.

\section{REFERENCES}

1. Simon MV. Neurophysiologic intraoperative monitoring of the vestibulocochlear nerve. J Clin Neurophysiol 2011;28:566-81.

2. Legatt $\mathrm{AD}$. Mechanisms of intraoperative brainstem auditory evoked potential changes. J Clin Neurophysiol 2002;19:396-408.

3. Martin WH, Stecker MM. ASNM position statement: Intraoperative monitoring of auditory evoked potentials. J Clin Monit Comput 2008;22:75-85.

4. Jewett DL, Romano MN, Williston JS. Human auditory evoked potentials: Possible brain stem components detected on the scalp. Science 1970;167:1517-8.

5. Levine RA, Ojemann RG, Montgomery WW, McGaffigan PM. Monitoring auditory evoked potentials during acoustic neuroma surgery. Insights into the mechanism of the hearing loss. Ann Otol Rhinol Laryngol 1984;93 (2 Pt 1):116-23.

6. James ML, Husain AM. Brainstem auditory evoked potential monitoring: When is change in wave $\mathrm{V}$ significant? Neurology 2005;65:1551-5.

7. Møller AR, Møller MB. Does intraoperative monitoring of auditory evoked potentials reduce incidence of hearing loss as a complication of microvascular decompression of cranial nerves? Neurosurgery 1989;24:257-63. 